\title{
Cambios tamaño-dependientes en la dieta de peces marinos y su estudio mediante análisis de isótopos estables
}

\author{
Manuela Funes'; Ana Laura LiberofF²; David E. Galván ${ }^{2, \bowtie}$ \\ 1. Universidad Nacional de la Patagonia San Juan Bosco, Puerto Madryn, Argentina. 2. Centro Nacional Patagónico \\ (CONICET), Puerto Madryn, Argentina.
}

\begin{abstract}
RESUMEN. Los peces sufren significativas modificaciones morfológicas a lo largo de su vida, como el cambio en la longitud relativa del tubo digestivo o el aumento de tamaño de boca, y algunas de ellas pueden derivar en cambios en el nivel trófico (NT). Estudiar este fenómeno es importante para entender la trofodinámica de cada especie, verificar los supuestos de los modelos de tramas tróficas estructurados por tallas y para monitorear el estado de una comunidad a través de los espectros de talla. En el presente trabajo se evaluaron los cambios en la posición trófica con respecto al tamaño corporal para cuatro especies de peces que habitan los arrecifes norpatagónicos, Pagrus pagrus, Diplodus argentus argentus, Pinguipes brasilianus y Acanthistius patachonicus. Aunque estas especies comparten los sitios de residencia y se ubican aproximadamente dentro del mismo nivel trófico (el nivel trófico varió entre 3.98 y 4.45), presentaron variaciones especie-específicas en los valores isotópicos con respecto al aumento de su tamaño corporal, que reflejarían comportamientos tróficos diferentes. Pagrus pagrus y A. patachonicus exhibieron un aumento en el nivel trófico correlacionado con el tamaño, mientras que $P$. brasilianus y $D$. argenteus no presentaron cambios en el nivel trófico en función del tamaño corporal. Estos resultados llaman la atención sobre la necesidad de contemplar la identidad de las especies en los modelos de cadena trófica estructurados por talla, sin suponer como generalizado el cambio alimentario talla-dependiente. Sin embargo los resultados apoyarían la hipótesis de que a más amplio el rango de crecimiento de un pez, mayor es la tendencia al aumento de nivel trófico conforme crece.
\end{abstract}

[Palabras clave: Nivel trófico, trofodinámica, crecimiento, ontogenia, arrecifes rocosos]

\begin{abstract}
Aвstract. Size-based dietary shifts in marine fishes assessed by stable isotopes analysis: Fish undergo significant morphological changes throughout their lives, like in the relative length of the digestive tube or the increase of mouth gape, and some of them can lead to changes in the trophic level. Studying these changes is important to understand fish trophodynamics, to verify the assumptions of size-structured trophic models and to assess community status by analyzing the size spectrum. In the present study we evaluated changes in the trophic positions of four reef-fish from northern Patagonia: Pagrus pagrus, Diplodus argentus argentus, Pinguipes brasilianus and Acanthistius patachonicus. Even though these four species share foraging areas and had similar trophic levels (trophic level ranged between 3.98 and 4.45), they exhibited specie-specific isotopic trends related to changes in body size that could be meaning different trophic behavior. While $P$. pagrus and A. patachonicus presented an increase in their trophic level positively correlated with body size, D. argenteus and $P$. brasilianus did not show any isotopic trend along ontogeny. These results highlight the importance of including the species identity in size-structured trophic models, as well as to acknowledge that size-based feeding behavior might not be ubiquitous. However, these results support the assumption that as the maximum body size of a species increases, there are more probabilities to increase trophic level with size.
\end{abstract}

[Keywords: Trophic level, trophodinamics; growth; ontogenetic changes; rocky reefs]

\section{INTRODUCCIÓN}

"El pez grande se come al más chico (y así al pobre el rico)" enuncia el popular refrán, cuyo fundamento resulta intuitivo. Sin embargo, tras estas palabras se esconde un fenómeno biológico mucho más complejo. Dado que los peces son organismos de crecimiento continuo y sufren significativas modificaciones en su historia de vida, se supone como característica común a la mayoría de los peces marinos (exceptuando herbívoros) que el aumento en la talla conlleva a un aumento en el nivel trófico (Jennings et al. 2001; Stergiou \& Karpouzi 2002). Dichos

\section{Editor asociado: Fernando Unrein}

cambios en la dieta en relación al crecimiento se pueden clasificar en tres grandes grupos: 1 ) los ontogénicos que resultan del desarrollo de un organismo, como por ejemplo variaciones del tubo digestivo o en la bioenergética del animal; 2) los que sólo son función de las limitaciones morfológicas de tamaño en la alimentación, como el aumento del tamaño de la boca; y 3) los inducidos por un cambio en el uso del hábitat, como las migraciones (Cohen et al. 1993; Reid et al. 2007; Sweeting et al. 2012). El presente trabajo se sitúa en el marco del segundo grupo, por lo que se espera que los cambios en la dieta se manifiesten como variaciones en el nivel trófico (Greenwood et 
al. 2010). De esta manera, los peces al crecer tendrían la capacidad de incorporar presas de mayor tamaño y aumentarían su posición en la trama trófica con una tendencia a la piscivoría (Jennings et al. 2001; 2002).

La existencia o no este tipo de variaciones en la dieta de los peces en función del tamaño corporal se ha puesto a prueba en numerosos trabajos, tanto para comprender el funcionamiento de los ecosistemas marinos (Deudero et al. 2004; Jennings et al. 2007) como para adoptar medidas de manejo apropiadas en pesquerías o áreas protegidas (e.g., Jennings et al. 2001). Algunas de las más exhaustivas revisiones de datos (Davenport \& Bax 2002; Jennings et al. 2002; Sweeting et al. 2012) sugieren que los cambios en la alimentación dependientes del tamaño son un fenómeno común en los peces marinos. Sin embargo, Galván et al. (2010) muestra que de 131 relaciones talla/nivel trófico publicadas, sólo el 36\% resultaron relaciones positivas, el $4 \%$ resultaron negativas y alrededor de un $60 \%$ fueron no significativas, adjudicando al menos el $50 \%$ de las relaciones no significativas a diseños experimentales poco potentes con muestras pequeñas o rangos de talla muy acotados. A estas diferencias en relación a la tendencia general se suman trabajos que muestran tendencias opuestas o inconsistentes dentro de una misma especie (Greenwood et al. 2010). No obstante lo expuesto, los cambios en el nivel trófico relacionados con el tamaño se siguen suponiendo una tendencia común a la mayoría de los peces, pero cuya intensidad varia entre y dentro de las especies, dependiendo de la complejidad de las trama trófica local y de la importancia y naturaleza de los subsidios de alimento que recibe de otras tramas tróficas (Polis \& Strong 1996). Estas variaciones en los resultados publicados ilustran la complejidad del fenómeno de cambio en el nivel trófico en función del crecimiento y la vigencia que exhibe su estudio ya que tiene implicancias tanto teóricas como prácticas. En este sentido, si el tamaño corporal constituye un parámetro aproximado del nivel trófico, el análisis del espectro de tallas capturado puede ser utilizado para evaluar posibles impactos de la pesquería sobre la estructura trófica de las comunidades y los ecosistemas marinos (Jennings et al. 2001). Por otra parte, la correcta descripción de los cambios en la dieta a lo largo del desarrollo, además de aportar al conocimiento general de las especies, es necesaria para describir su "hábitat esencial" (i.e. aguas y sustratos que aseguren la alimentación, crecimiento hasta la madurez y reproducción; Stal et al. 2007) y dar herramientas para una planificación efectiva de medidas de manejo ecosistémicas o espaciales, como la delimitación de áreas protegidas (Blyth-Skyrme et al. 2006; Gunderson et al. 2008).

Una herramienta robusta y crecientemente utilizada para estimar el nivel trófico de un organismo es el Análisis de Isótopos Estables (AIE) (Boecklen et al. 2011). El AIE utiliza las diferencias naturales en las abundancias de ${ }^{15} \mathrm{~N} \mathrm{y}{ }^{14} \mathrm{~N}$, y de ${ }^{13} \mathrm{C}$ y ${ }^{12} \mathrm{C}$ entre los tejidos de los consumidores y sus recursos alimentarios (DeNiro \& Epstein 1978). Estas diferencias se adjudicanprincipalmentealacaptaciónselectiva de compuestos ${ }^{13} \mathrm{C}$ durante la digestión, a la pérdida preferencial del ${ }^{12} \mathrm{CO}_{2}$ en la respiración, así como una excreción preferencial del isótopo ${ }^{14} \mathrm{~N}$, llevando a un enriquecimiento relativo en ${ }^{15} \mathrm{~N} \mathrm{y}{ }^{13} \mathrm{C}$ entre el consumidor y su presa, denominado fraccionamiento trófico (Fry 2006). El fraccionamiento de ${ }^{15} \mathrm{~N}$ en cada nivel trófico $\left(\Delta \delta^{15} \mathrm{~N}\right)$ de los peces marinos es amplio, $\Delta \delta^{15} \mathrm{~N}$ $\approx 3.2 \%$ (Sweeting et al. 2007a), permitiendo la estimación del nivel trófico con un alto grado de certeza (Post 2002). El ${ }^{13} \mathrm{C}$ tiene un fraccionamiento más pequeño que e ${ }^{15} \mathrm{~N}$, siendo en peces $\Delta \delta^{13} \mathrm{C} \approx 1.5 \%$ (Sweeting et al. 2007b). Dado que el $\delta^{13} \mathrm{C}$ es muy variable en la base de la cadena trófica (productores primarios), ha sido principalmente utilizado para aportar información acerca de las diferentes fuentes de alimento en los consumidores superiores. En este sentido, las algas bentónicas a menudo muestran valores más altos de $\delta^{13} \mathrm{C}$ que las algas planctónicas (France 1995) por lo que la relación entre los isótopos estables de C ha sido muy útil para determinar diferencias entre cadenas tróficas bentónicas y pelágicas.

Dentro del marco teórico y metodológico enunciado,elpresente trabajoproponeestudiar los cambios tróficos tamaño-dependientes en cuatro especies de peces de arrecife: Pagrus pagrus (Linnaeus, 1758) (Besugo), Diplodus argentus argentus (Valenciennes 1830) (Sargo), Pinguipes brasilianus (Cuvier 1829) (Turco) y Acanthistius patachonicus (Jenyns 1840) (Mero), utilizando la composición isotópica de estas especies como descriptor de su nivel trófico.

\section{MATERIALES Y MÉTODOS}

\section{Especies en estudio}

En el conjunto de peces de arrecifes que habitan el sur del golfo San Matías, las especies seleccionadas son las más abundantes y conspicuas (Galván et 
al. 2009a; Irigoyen \& Galván 2010); por lo que su comportamiento trófico puede considerarse representativo de las grandes tendencias tróficas dentro del ensamble.

El besugo, Pagrus pagrus, es un espárido que se distribuye ampliamente en el Océano Atlántico; ocurriendo en la costa americana desde Nueva York $\left(41^{\circ} \mathrm{N}\right)$ hasta los golfos norpatagónicos $\left(46^{\circ} \mathrm{S}\right)$, entre los $10 \mathrm{~m}$ y los $80 \mathrm{~m}$ de profundidad (Galván et al. 2005). Presenta gran valor comercial a escala mundial y por su sobrepesca ha sido declarado especie "En peligro" por la Unión Internacional para la Conservación de la Naturaleza (UICN, Huntsman 1996). En Argentina es capturado principalmente en la costa bonaerense y en menor escala en el Golfo San Matías. Se crecimiento es lento y alcanza la edad de 16 años, estimándose una edad de tres años (talla $\approx 26 \mathrm{~cm}$ ) para la madurez sexual. El besugo puede superar los $60 \mathrm{~cm}$ (Froese \& Pauly 2013), aunque en los golfos norpatagónicos el largo máximo $\left(L_{\max }\right)$ observado no supera los 40 cm (Galván et al. 2005; Irigoyen \& Galván 2010). Es predador de los organismos asociados a los arrecifes norpatagónicos, alimentándose principalmente de invertebrados, como poliquetos y crustáceos, y de peces pequeños (Brankevich et al. 1990; Irigoyen \& Galván 2010).

El Sargo, Diplodus argenteus argenteus, presenta una distribución más amplia aún, desde Florida EEUU $\left(30^{\circ} \mathrm{N}\right)$ hasta el norte del golfo San Jorge $\left(46^{\circ} \mathrm{S}\right)$. Es una especie de hábitos semi-pelágicos que se asocia principalmente a la franja costera y es raro a profundidades mayores a $15 \mathrm{~m}$. Se alimenta en general de invertebrados chicos como bivalvos, lapas, quitones, cangrejos. Además se sabe que incorpora grandes cantidades de algas en su dieta, sin la certeza aún de si se alimenta de ellas o su ingesta es producto de la depredación sobre los invertebrados asociados a éstas. La talla máxima para la zona es de $34 \mathrm{~cm} L_{F}$ (Galván et al. 2005) aunque alcanza los $38 \mathrm{~cm}$ en aguas de Brasil (David et al. 2005).

El turco, Pinguipes brasilianus, es un pinguipedido que se distribuye desde el sur de Brasil $\left(24^{\circ} \mathrm{S}\right)$, hasta el norte del Golfo San Jorge en la Patagonia Argentina $\left(46^{\circ} \mathrm{S}\right)$. Se encuentra principalmente en aguas someras, desde el intermareal hasta los $60 \mathrm{~m}$ (Cousseau \& Perrotta 2000). Presenta una talla mediana, llega a los $40 \mathrm{~cm}$ y $1 \mathrm{~kg}$ de peso (Irigoyen \& Galván 2010). Se lo señala como consumidor de invertebrados bentónicos como poliquetos, bivalvos, cangrejos y erizos, aunque ocasionalmente suele incorporar peces a su dieta (Galván et al. 2009b).

El Mero, Acanthistius patachonicus, es una especie muy abundante entre los $38^{\circ}$ y $45^{\circ} \mathrm{S}$ con una distribución total que abarca desde San Pablo, Brasil $\left(23^{\circ} \mathrm{S}\right)$ hasta el sur del golfo San Jorge, $\left(48^{\circ} \mathrm{S}\right)$; y de la costa hasta los $130 \mathrm{~m}$, en registros de máxima profundidad. Es una de las especies preferidas para la pesca artesanal, por su abundancia, tamaño y el sabor de su carne. Alcanza una talla máxima en la zona de $65 \mathrm{~cm}$. Los principales ítems presa descriptos para esta especie son cangrejos, peces y poliquetos (Galván et al. 2009b).

\section{Toma y preparación de las muestras}

El muestreo se llevó a cabo en la playa Puerto Lobos, ubicada en el Golfo San Matías $\left(42^{\circ} \mathrm{S} 65^{\circ} \mathrm{O}\right.$, Figura 1) en Febrero de 2012. Los ejemplares se capturaron utilizando elementos de pesca submarina y líneas de anzuelos, intentando obtener una distribución de tallas lo más continua, uniforme y completa posible, según las recomendaciones de Galván et al. (2010). Todas las capturas se realizaron en el mismo sitio de pesca y el mismo día para cada especie, minimizando las fuentes de variación no relacionados directamente con la ontogenia (e.g. estacionalidad, migraciones). Los peces se sacrificaron a bordo de la embarcación, con el fin de atenuar el sufrimiento; y se almacenaron en conservadoras con hielo, asegurando la óptima y correcta preservación de las muestras (Carabel et al. 2006). Los ejemplares se procesaron en el laboratorio la misma tarde de la fecha de captura.

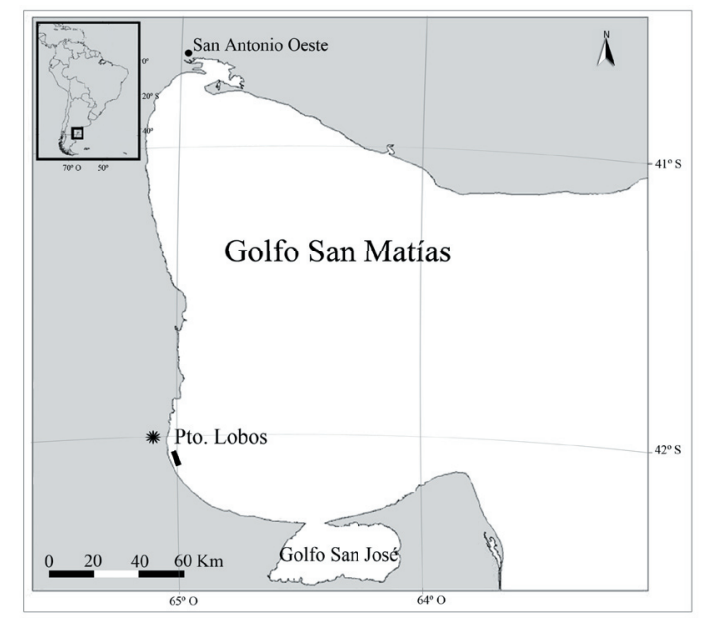

Figura 1. Mapa del sitio de estudio, Playa Puerto Lobos, Golfo San Matías, Patagonia Argentina. El rectángulo negro muestra la ubicación del arrecife donde se realizó el muestreo.

Figure 1. Study site. Puerto Lobos beach, San Matías Gulf, Patagonia Argentina. The rectangle shows the location of the reef were the sampling was conducted.

En el laboratorio se pesó y midió a los individuos, tomando el largo total $\left(L_{T}\right)$ para las especies con cola truncada, turco y mero; y el largo de furca $\left(L_{F}\right)$ para las especies con cola escotada, besugo y sargo, con el propósito de minimizar los errores de medición. De cada animal se extrajo una fracción de músculo dorsal blanco (Pinnegar \& Polunin 1999) y se lo secó a $60^{\circ} \mathrm{C}$ hasta logar un peso estabilizado. Se molieron manualmente por separado y se almacenaron en un desecador para su posterior utilización. Las muestras individuales de aproximadamente 1.25 $\mu \mathrm{g}$ de fueron enviadas al laboratorio de isótopos estables de la Universidad Davis de California para su determinación analítica. Los resultados 
del espectrómetro de masa se informaron como valores de $\delta$ :

$\delta X=[(\mathrm{R}$ muestra / $\mathrm{R}$ estándar $)$-1] x 1000, donde $X$ es ${ }^{13} \mathrm{C}$ o ${ }^{15} \mathrm{~N}$, $\mathrm{R}$ es la relación ${ }^{13} \mathrm{C} /{ }^{12} \mathrm{C}$ o ${ }^{15} \mathrm{~N} /{ }^{14} \mathrm{~N}$ correspondiente; como estándar se utilizó el $\mathrm{N}^{2}$ atmosférico para el $\mathrm{N}$ y la belemita de Viena Peedee para el C.

\section{Análisis de los datos}

Para evaluar la representatividad del muestreo realizado, se calculó la mínima amplitud del cambio en unidades de nivel trófico que el mismo permitiría detectar para cada especie, con una potencia de 0.80 (i.e., la sensibilidad del muestreo para detectar un determinado cambio en el nivel trófico de una especie, a lo largo de su rango de talla). Para ello se relativizó la talla mínima y máxima muestreada en cada una de las especies con respecto al propio $\mathrm{L}_{\max }$ según la fórmula: $\Delta \ln \left(L_{\max }\right)=\ln \left(L_{\max o b s} / L_{\max }\right)$ - ln $\left(L_{\text {minobs }} / L_{\max }\right)$ (Galván et al. 2010), donde los largos corresponden a $L_{T}$ o $L_{F}$ según la especie, $L_{\max }$ es el largo máximo registrado para la zona y $L_{\text {maxobs }}$ y $L_{\text {minobs }}$ son los largos máximos y mínimos del presente muestreo. Luego se ingresó con el valor $\Delta \log \left(L_{\max }\right)$ al gráfico generado por la función power_TL.dn (Galván et al. 2010) escrita para el software estadístico R (R Development Core Team, 2010). En función del tamaño muestral y el $\Delta \ln \left(L_{\max }\right)$ calculado se obtuvo entonces la mínima amplitud de cambio trófico detectable para cada especie. Dado que un alto porcentaje lipídico en las muestras puede distorsionar los valores de $\delta^{13} \mathrm{C}$ respecto de lo esperado por enriquecimiento trófico, se evaluó el contenido de lípidos a partir de las relaciones C(ug)/N(ug) (Logan et al. 2008). Se aplicó una corrección aritmética (Sweeting et al. 2006, con la modificación de Greenwood et al. 2010) para los valores de $\delta^{13} \mathrm{C}$ de las especies con muestras que tuvieron relaciones $\mathrm{C} / \mathrm{N}>3.5$ (ver resultados).

Para poner a prueba si existen cambios en el nivel trófico respecto del tamaño corporal se realizaron análisis de regresión (Crawley 2007) sobre los valores $\delta^{15} \mathrm{~N}$ y $\delta^{13} \mathrm{C}$ vs. la talla y vs. el peso en cada una de las especies. Aquellas especies que mostraron tendencias significativas y similares (positivas o negativas en función de los valores $\delta^{15} \mathrm{~N}$ ) se combinaron en un modelo general. Se incluyó la variable peso que, si bien es una medida de tamaño con mayor variación que la talla respecto de la edad (un pez puede perder peso pero no reducir su largo), es un buen descriptor de posibles efectos alométricos como las relaciones no lineales entre el tamaño de boca y el largo del cuerpo (Karpouzi \& Stergiou 2003). Las regresiones se probaron con y sin transformación logarítmica para las variables de tamaño, y se eligió la alternativa que explicó una mayor fracción de la varianza observada (i.e., mayor valor de coeficiente de determinación muestral, $\mathrm{r}^{2}$ ). A posteriori se evaluaron los supuestos de la regresión normal mediante el análisis de sus residuos, generando una nueva regresión entre los valores esperados y los residuales de cada modelo, bajo la hipótesis nula de que no existe una tendencia en los residuales respecto del valor predicho y siguiendo el protocolo descripto por Jiao et al. (2004). Mediante el gráfico diagnóstico de residuales vs. la influencia de cada punto (Leverage) se evaluó la existencia de puntos con mayor peso en la estimación de los parámetros, y se probó el cambio de significancia de cada regresión excluyendo de a uno a los puntos más influyentes, según el protocolo descripto por Crawley (2007). Se estimó la posición trófica de cada especie para ubicarlas en la trama trófica, y compararlas entre sí, mediante la ecuación: NT $=\lambda+\left(\delta^{15} \mathrm{~N}\right.$ $\left.-\delta^{15} N_{\text {base }}\right) / \Delta \delta^{15} \mathrm{~N}$ (Post et al. 2000)

Donde $\lambda=2$ es la posición trófica del organismo utilizado para estimar el valor $\delta^{15} \mathrm{~N}_{\text {base }} \mathrm{y} \Delta \delta^{15} \mathrm{~N}=3.2 \mathrm{es}$ el valor de fraccionamiento trófico según Sweeting (2007a). Se utilizó como $\delta^{15} \mathrm{~N}_{\text {base }}$ al promedio entre los valores de $\delta^{15} \mathrm{~N}$ del gasterópodo herbívoro Tegula patagonica, el bivalvo filtrador Aulacomya atra y copépodos del plancton (entre $280 \mu \mathrm{m}$ y $420 \mu \mathrm{m}$ ), muestreados en el área en abril de 2009. Además se comparó el nicho isotópico mediante el cálculo del tamaño y la posición de la elipse estándar (SEA), la cual describe en forma univariada la variación en los ejes $\delta^{15} \mathrm{~N}$ y $\delta^{13} \mathrm{C}$ (Jackson et al. 2011). Todos los análisis estadísticos se realizaron utilizando el software estadístico R V.2.11.1 (R Development Core Team, 2010); el cálculo de las elipses estándar se realizó con el paquete SIAR (Jackson et al. 2011) para el software R.

\section{Resultados}

Se muestrearon 22 individuos de besugo, 18 de sargo 13 de turco y 16 de mero, cubriendo entre el $38 \%$ y el $60 \%$ de sus distribuciones de tallas observadas en la zona de estudio (Tabla 1). Este muestreo proporcionaría una capacidad teórica de detección de $\Delta \mathrm{NT} \geq$ 0.15 para el besugo, $\Delta \mathrm{NT} \geq 0.5$ para el sargo y el mero, y $\Delta \mathrm{NT} \geq 0.25$ para el turco. Sólo las especies de espáridos, besugo y sargo, presentaron relaciones $\mathrm{C} / \mathrm{N}>3.5$ (Tabla $1)$, indicando que contenían cantidades significativas de lípidos. Por esta razón se aplicó una corrección aritmética a los valores de $\delta^{13} \mathrm{C}$ de estas dos especies con el fin de minimizar cualquier variación no relacionada con los cambios tróficos.

El besugo y el mero mostraron una relación significativa entre el valor de $\delta^{15} \mathrm{~N}$ y el tamaño de los individuos, aumentando el nivel trófico con la talla (Figura 2) y el peso (Tabla 2). De igual manera los valores de $\delta^{13} \mathrm{C}$ aumentaron en relación al tamaño corporal, tanto en talla (Figura 2) como en peso, en forma significativa (Tabla 2). En el caso del turco y el sargo no se observaron tendencias de ninguna de las señales isotópicas con respecto al tamaño que 

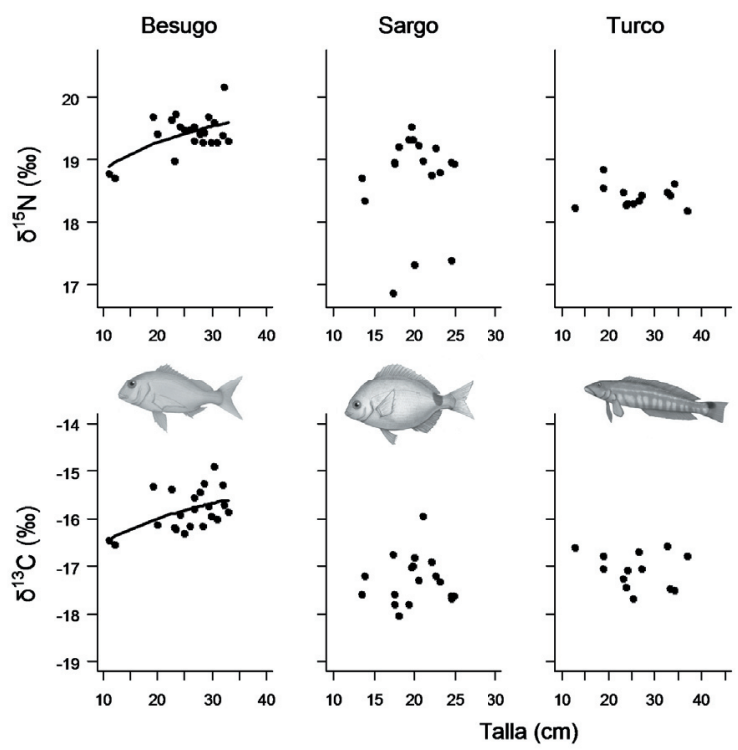

pudieran indicar un cambio en la alimentación debido al crecimiento; siendo las regresiones no significativas tanto para los valores de $\delta^{15} \mathrm{~N}$ como de $\delta^{13} \mathrm{C}$ (Tabla 2). La significancia de las regresiones fue similar con la aplicación o no de la transformación logarítmica a las variables de tamaño corporal, aunque se obtuvieron mejores ajustes (mayores valores de $\mathrm{r}^{2}$ ) para los datos transformados. Las variables peso y talla mostraron similar capacidad explicativa del nivel trófico para todas las especies (ver coeficientes de regresión en Tabla 2). La combinación de los datos de mero y besugo mostró una relación positiva y significativa entre los valores de $\delta^{15} \mathrm{~N}$ en función de la talla (Tabla 2 y Figura 3) y en función del peso (Tabla 2). Ambas especies mostraron valores similares de $\delta^{15} \mathrm{~N}$ en el rango en que sus tamaños corporales se solapan (Figura 3), lo cual se pone en evidencia en la alta significancia y buen ajuste del análisis de regresión (Tabla 2), que en este caso fueron más altos para los datos sin transformación logarítmica. En relación a la validación de los modelos, el análisis de los residuales no

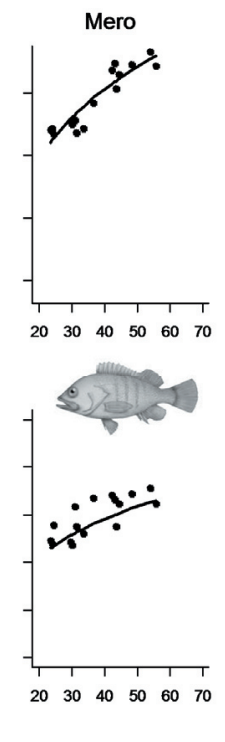

Figura 2. Valores de $\delta^{15} \mathrm{~N}$ y $\delta^{13} \mathrm{C}$ de las cuatro especies estudiadas en relación a la talla (largo de furca $\left(L_{F}\right)$ para el besugo y el sargo y largo total $\left(L_{T}\right)$ para el turco y el mero). Las líneas muestran los valores $\delta \mathrm{X}$ predichos para la variable $\ln$ (Talla) por los modelos de regresión generados a partir de las regresiones significativas.

Figure 2. Relationship between $\delta^{15} \mathrm{~N}$ and $\delta^{13} \mathrm{C}$ values and fish size, fork length $\left(L_{\digamma}\right)$ for besugo and sargo and total length $\left(L_{T}\right)$ for turco and mero. Lines represent $\delta X$ predicted values for the variable $\ln$ (Size) when regression models were significant.

mostró tendencias significativas $\left(\mathrm{H}_{0}: b=0 ; P>\right.$ $0.05)$ lo que permitió suponer que se cumplió con los supuestos del método. Los gráficos de diagnóstico y el reajuste de los modelos permitieron descartar la presencia de puntos influyentes en las regresiones significativas. La ausencia de puntos influyentes fue de particular importancia en el besugo para descartar que los dos individuos de menor talla sean los responsables de forzar la tendencia informada (Figura 2).

Las cuatro especies se ubicaron dentro del cuarto nivel trófico (Figura 4). Los niveles tróficos promedio fueron, para el turco 3.98 (desvío estándar, DE 0.05), para el sargo 4.07 (DE 0.23), para el besugo 4.30 (DE 0.10) y para el mero 4.45 (DE 0.15). Mientras que el nicho isotópico del turco estuvo totalmente superpuesto con el del sargo, el del resto de las especie se diferenció claramente sin mostrar solapamientos (Figura 4). El nivel trófico del mero y el besugo fue mayor que el de las otras dos especies. Por otra parte, el sargo fue la especie que mostró mayor amplitud de nicho

Tabla 1. Datos de tamaño corporal (peso, talla y porcentaje del rango de tallas), composición elemental (relación C: $\mathrm{N})$ e isotópica $\left(\delta^{13} \mathrm{C}\right.$ y $\left.\delta^{15} \mathrm{~N}\right)$ obtenidos en las 4 especies estudiadas. Se presenta también el tamaño de muestra para cada especie. Los valores de talla del besugo y sargo corresponden al largo de furca (LF), mientras que en el caso del turco y mero se refieren al largo total (LT).

Table 1. Information on body size (length, weight and percentage of size range covered), elemental (C:N) and isotopic $\left(\delta^{13} \mathrm{C}\right.$ and $\left.\delta^{15} \mathrm{~N}\right)$ composition. Table also shows sample size for each species. Lengths informed are fork length $\left(L_{F}\right)$ for besugo and sargo and total length $\left(L_{T}\right)$ for the other two species.

\begin{tabular}{lcccccc}
\hline & $\begin{array}{c}\text { Peso }(\mathrm{gr}) \\
\text { Min - Max }\end{array}$ & $\begin{array}{c}\text { Talla }(\mathrm{cm}) \\
\text { Min - Max }\end{array}$ & $\begin{array}{c}\text { Rango tallas } \\
(\%)\end{array}$ & C:N & $\delta^{13} \mathbf{C}(\%)$ & $\delta^{15} \mathbf{N}(\%)$ \\
\hline Besugo (22) & $40-775$ & $10.9-33$ & 55 & $3.4 \pm 0.13$ & $-16.39 \pm 0.41$ & $19.41 \pm 0.31$ \\
Sargo (18) & $70-442$ & $13.5-24.8$ & 38 & $3.8 \pm 0.35$ & $-18.45 \pm 0.60$ & $18.71 \pm 0.75$ \\
Turco (16) & $32-560$ & $12.8-36.8$ & 60 & $3.15 \pm 0.05$ & $-17.19 \pm 0.34$ & $18.42 \pm 0.18$ \\
Mero (13) & $190-2880$ & $23.5-55.7$ & 50 & $3.18 \pm 0.03$ & $-16.22 \pm 0.43$ & $19.89 \pm 0.48$ \\
\hline
\end{tabular}


Tabla 2. Resultados de las regresiones entre las variables de tamaño corporal y las composiciones isotópicas. La tabla muestra los parámetros de las regresiones ( $a=$ ordenada al origen y $b=$ pendiente), sus correspondientes desvíos estándares $(D E)$, el coeficiente de determinación muestral $\left(r^{2}\right)$ y la significancia de la regresión $(P ; \mathrm{H} 0 b=0)$.

Table 2. Results of the regressions performed between body-size variables and isotopic composition. Table shows regression parameters ( $a=$ intercept and $b=$ slope), their standard deviations $(D E)$, correlation coefficient $\left(r^{2}\right)$ and significance level $(P ; \mathrm{H} 0 b=0)$.

\begin{tabular}{lcccccc}
\hline & $b$ & $D E$ & $a$ & $D E$ & $r^{2}$ & $P$ \\
\hline Besugo & & & & & & \\
$\delta^{15} \mathrm{~N}$ vs. $\ln \left(L_{F}\right)$ & 0.625 & 0.197 & 17.410 & 0.635 & 0.333 & 0.005 \\
$\delta^{15} \mathrm{~N}$ vs. $\ln ($ Peso $)$ & 0.243 & 0.074 & 17.993 & 0.437 & 0.349 & 0.003 \\
$\delta^{13} \mathrm{C}$ vs. $\ln \left(L_{F}\right)$ & 0.771 & 0.289 & -18.304 & 0.929 & 0.262 & 0.014 \\
$\delta^{13} \mathrm{C}$ vs. $\ln ($ Peso $)$ & 0.329 & 0.104 & -7.759 & 0.616 & 0.303 & 0.005 \\
Sargo & & & & & & \\
$\delta^{15} \mathrm{~N}$ vs. $\ln \left(L_{F}\right)$ & 0.319 & 1.064 & 17.763 & 3.174 & 0.006 & 0.768 \\
$\delta^{15} \mathrm{~N}$ vs. $\ln ($ Peso $)$ & 0.120 & 0.358 & 18.073 & 1.923 & 0.007 & 0.742 \\
$\delta^{13} \mathrm{C}$ vs. $\ln \left(L_{F}\right)$ & 0.179 & 0.707 & -17.822 & 2.109 & 0.004 & 0.803 \\
$\delta^{13} \mathrm{C}$ vs. $\ln ($ Peso $)$ & 0.090 & 0.238 & -17.770 & 1.276 & 0.009 & 0.709 \\
Turco & & & & & & \\
$\delta^{15} \mathrm{~N}$ vs. $\ln \left(L_{T}\right)$ & -0.028 & 0.610 & 18.517 & 0.610 & 0.002 & 0.884 \\
$\delta^{15} \mathrm{~N}$ vs. $\ln ($ Peso $)$ & -0.030 & 0.063 & 18.585 & 0.338 & 0.020 & 0.642 \\
$\delta^{13} \mathrm{C}$ vs. $\ln \left(L_{T}\right)$ & -0.480 & 0.321 & -15.650 & 1.039 & 0.168 & 0.164 \\
$\delta^{13} \mathrm{C}$ vs. $\ln ($ Peso $)$ & -0.116 & 0.113 & -16.583 & 0.609 & 0.086 & 0.331 \\
Mero & & & & & & \\
$\delta^{15} \mathrm{~N}$ vs. $\ln \left(L_{T}\right)$ & 1.599 & 0.176 & 14.167 & 0.632 & 0.854 & $<0.001$ \\
$\delta^{15} \mathrm{~N}$ vs. $\ln ($ Peso $)$ & 0.534 & 0.059 & 16.366 & 0.393 & $0.853<0.001$ \\
$\delta^{13} \mathrm{C}$ vs. $\ln \left(L_{T}\right)$ & 1.152 & 0.267 & -20.346 & 0.958 & $0.571<0.001$ \\
$\delta^{13} \mathrm{C}$ vs. $\ln ($ Peso $)$ & 0.389 & 0.088 & -18.787 & 0.586 & $0.581<0.001$ \\
Besugo y Mero & & & & & & \\
$\delta^{15} \mathrm{~N}$ vs. $\operatorname{Talla}$ & 0.039 & 0.003 & 18.416 & 0.127 & $0.730<0.001$ \\
$\delta^{15} \mathrm{~N}$ vs. $\operatorname{Peso}$ & $6.1 \mathrm{e}-04$ & $7.2 \mathrm{e}-05$ & 19.210 & 0.064 & $0.668<0.001$ \\
\hline
\end{tabular}

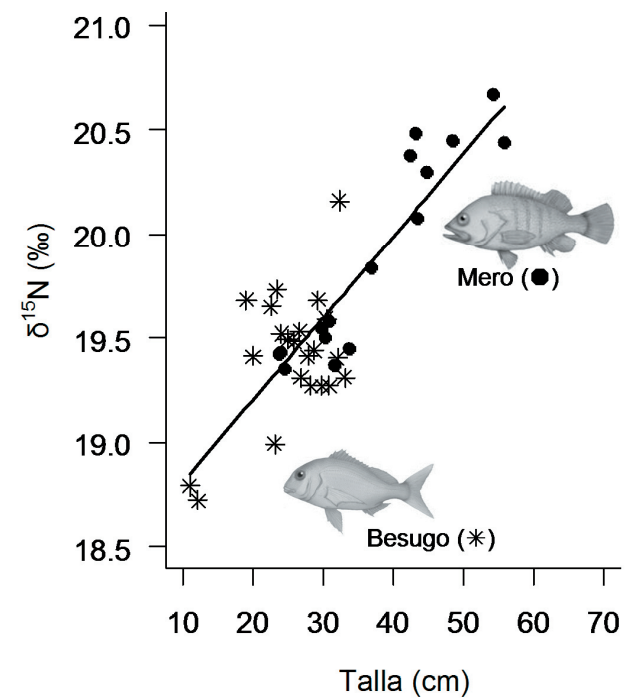

Figura 3. Valores de $\delta^{15} \mathrm{~N}$ y $\delta^{13} \mathrm{C}$ para el besugo y el mero en relación a la talla, $L_{F}$ para el besugo y $L_{T}$ para el mero. La línea muestra los valores predichos por el modelo de regresión ajustado combinando los datos de ambas especies.

Figure 3. Relationship between $\delta^{15} \mathrm{~N}$ and $\delta^{13} \mathrm{C}$ values and fish size for besugo and mero, $L_{F}$ for besugo and $L_{T}$ for mero. Line shows predicted values by the regression model fitted joining both species' data.

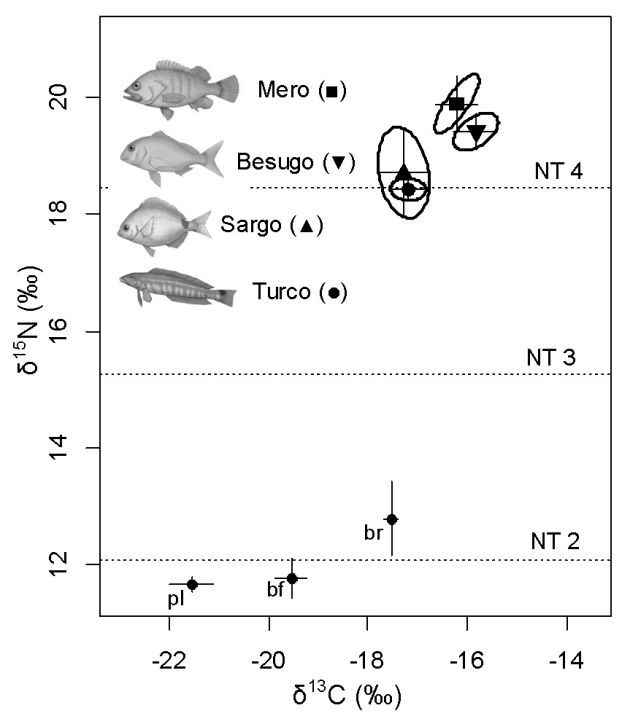

Figura 4. Representación de la posición trófica relativa de los peces estudiados con respecto a la línea de base en la comunidad. La figura muestra las firmas isotópica medias (valores $\delta^{13} \mathrm{C}$ vs $\delta^{15} \mathrm{~N}$ ) y las elipses estándar de los peces estudiados y las firmas isotópica medias de los organismos del segundo nivel trófico (pl: zooplancton, copépodos; bf: bentónico filtrador, Aulacomya atra y br: bentónico raspador herbívoro, Tegula patagonica). Las líneas de puntos dividen los niveles tróficos (NT) tomado como fraccionamiento trófico del $\mathrm{N}$ el valor $\Delta \delta^{15} \mathrm{~N}=3.2$.

Figure 4. Relative trophic level of the four fish studied in relation to the community baseline. Figure shows mean isotopic signatures ( $\delta 15 \mathrm{~N}$ and $\delta 13 \mathrm{C}$ values) and standard ellipses of fish species and mean isotopic signatures of the organism used to describe the second trophic level (pl: zooplankton, copepods; df: benthonic filter feeder, Aulacomya atra and br: benthonic grasser, Tegula patagonica). Dotted lines divide plot in trophic levels (NT) assuming trophic fractionation as $\Delta^{15} \mathrm{~N}=3.2$.

isotópico de acuerdo a las áreas de las elipses que describen su variación isotópica (Figura 4 , SEA sargo $=1.22$, mero $=0.42$, besugo $=0.38$ $\mathrm{y}$ turco $=0.21)$.

\section{DISCUSIÓN}

En el presente trabajo se observó que mientras que el besugo y el mero exhibieron un aumento de los valores de $\delta^{15} \mathrm{~N}$ y $\delta^{13} \mathrm{C}$ conforme el individuo crece, en el turco y el sargo la variación en estos valores no estuvo ligada al tamaño corporal dentro del rango de tallas muestreado. Por lo tanto, en coincidencia con lo propuesto para otros peces de arrecife, por ejemplo serránidos carnívoros (Deudero et al. 2004; Greenwood et al. 2010), el besugo y el mero presentarían un cambio en la alimentación a medida que crecen. La relación significativa entre el tamaño corporal y el nivel trófico se mantuvo aún combinando los datos para ambas especies, observándose una similitud en los valores de $\delta^{15} \mathrm{~N}$ donde 
las tallas se solaparon. Este resultado apoya parcialmente la hipótesis de que el tamaño corporal sería un buen descriptor de nivel trófico independientemente de la filiación específica (Jennings et al. 2007). Sin embargo, los resultados obtenidos para el turco y el sargo contradicen esta afirmación, indicando la importancia de no suponer que la mayoría de las especies de peces carnívoros $(\mathrm{NT} \geq 4)$ tienden a alimentarse de presas con mayor nivel trófico a medida que crecen. No obstante, dado que el mero y el besugo presentan mayores $L_{\max }$ que el turco y el sargo, nuestros resultados apoyarían el razonamiento de que a mayor amplitud de rango de crecimiento de un pez a lo largo de su vida, mayor cambio en sus habilidades tróficas y en consecuencia aumentan las probabilidades de exhibir una tendencia al aumento de nivel trófico conforme crece.

Si bien se interpretó al cambio en las señales isotópicas como cambios en el nivel trófico, existe la posibilidad de que otras variaciones, por ejemplo cambio en el tipo de presas, influyan en la variación de las señales isotópicas a lo largo de la ontogenia. Para el caso del besugo, los resultados indican que esta especie aumentaría su nivel trófico cerca de medio nivel a lo largo de su vida $(0.45$ nivel trófico según la talla). Por otra parte la magnitud del cambio en $\delta^{13} \mathrm{C}$ con respecto al tamaño (1.18\%) fue cerca del doble de la esperada por un aumento de medio nivel trófico $\left(\Delta \delta^{13} \mathrm{C} \approx 1.5\right)$. Esto último estaría vinculado con el uso de diferentes fuentes de energía a lo largo de su ontogenia, incorporando probablemente una mayor proporción de presas con una trama trófica basada en macroalgas bentónicas a medida que crece y disminuyendo el consumo de presas que se alimentan de una trama trófica a base de fitoplancton (Figura 4). Otro punto importante es que, si bien el muestreo no logró la uniformidad esperada en la distribución de tallas, observándose una discontinuidad entre los $13 \mathrm{~cm}$ y $18 \mathrm{~cm}$, sí se logró una buena representatividad de adultos y juveniles. Por esta razón, sumado a que todos los individuos fueron capturados en el mismo lugar y día, se discute el cambio observado en el besugo como un fenómeno gradual y no como un cambio más abrupto resultante de un cambio de hábitat y/o ligado a la ontogenia.

Por su parte, el mero presentó el rango de tallas absolutas más amplio de tamaño muestreado, permitiendo identificar cambios sutiles en el aumento de nivel trófico. Al alcanzar tallas tanto más grandes que las otras especies estudiadas, las capacidades alimenticias del mero cambian notablemente (tamaño de boca, velocidad de nado) y permiten establecer cambios más marcados en el nivel trófico en el cual se alimentan. Cambios en la dieta con una tendencia al aumento del nivel trófico en los individuos más grandes ya han sido documentados para esta especies (Galván et al. 2009b), aumentando la proporción de cefalópodos que incorporan a su dieta, y disminuyendo la proporción de cangrejos (Goldstein \& Cousseau 1987). Según el modelo de regresión estimado para esta especie, el cambio trófico a lo largo de la vida sería de alrededor de un nivel trófico (1.15 nivel trófico según la talla).

En el sargo y en el turco no se encontraron cambios en la posición trófica relacionados con el aumento de tamaño. Esto reflejaría que ambas especies mantienen una dieta similar a lo largo de su vida, aunque las relaciones de tamaño predador-presa probablemente cambien. Si bien el sargo fue la especie con menor amplitud de tallas cubierto se muestreó un $n$ suficiente como para detectar cambios ecológicamente significativos (sensu Galván et al. 2010); además estos resultados son congruentes con lo informado para otras cuatro especies del género Diplodus (Deudero et al. 2004). Por otro lado, en esta especie se observó gran variabilidad de las marcas de $\delta^{15} \mathrm{~N}$ y $\delta^{13} \mathrm{C}$ al aumentar el tamaño, reflejándose esto en DE más altos que las otras especies (Figura 3 y Tabla 2), lo que implicaría cierta variación interindividual e independiente del tamaño en el tipo de presas seleccionadas. Se ha observado que esta especie ingiere grandes cantidades de algas, pero se desconoce si son incorporadas o no a su organismo. Teniendo en cuenta que el nivel trófico promedio fue 4, podría postularse que las algas no aportan a la nutrición de esta especie, siendo ingeridas pero no incorporadas al organismo.

El turco fue la especie con menor número de individuos capturados, el rango de tallas fue el más alto de todos y tuvo una distribución regular de tallas, incrementando la potencia del análisis y la confiabilidad en los resultados. Esta especie, en contraposición al sargo, tendría menor variación intraindividual en su dieta, observado en los bajos DE de los valores de $\delta^{15} \mathrm{~N}$ y $\delta^{13} \mathrm{C}$ (Tabla 1$)$. Una visión comparativa de las cuatro especies muestra que aquellas con menor nivel trófico, el turco y el sargo, no presentan tendencias a aumentar su nivel trófico con el tamaño 
corporal mientras que sí lo hacen el besugo y el mero, que mostraron un nivel trófico mayor. Los niveles tróficos para el mero y el besugo fueron mayores que para el turco y el sargo (Figura 4) lo que podría reflejar una posible tendencia hacia la piscivoría por parte de las dos primeras especies. Sin embargo, estas diferencias fueron relativamente pequeñas y las cuatro especies se situaron dentro del cuarto nivel trófico, lo cual las ubica dentro del grupo de los mesocarnívoros.

Si bien la relación entre la agrupación por gremios tróficos de los predadores y la morfología del cuerpo es un área de intenso trabajo (Sibbing \& Nagelkerke 2000; Albouy et al. 2011), al momento se han publicado sólo unos pocos estudios que comparen la trofodinámica de diversas especies en relación a sus características anatómicas. En este sentido, Karpouzi \& Stergiou (2003) relacionan la pendiente de aumento de la apertura de boca con el crecimiento corporal y proponen que especies como Zeus faber o Merluccius merluccius, cuya boca crece rápidamente en función de la talla corporal, aumentan su nivel trófico al crecer; mientras que especies como Pagellus acarne, cuya boca crece más lentamente con respeto a la talla, no modifican su nivel trófico al crecer. Sin embargo, otros trabajos afirman que las características de la boca no son suficiente para describir las relaciones de tamaño predadorpresa (Scharf et al. 2000; Bachillier \& Irigoien 2013). En este sentido es clara la importancia de desarrollar estudios que correlacionen la trofodinámica de diversas especies de peces con variables como el tamaño y la posición de la boca junto a otras como la forma y área de la cola, o la relación entre el ancho del pedúnculo caudal y el ancho corporal, que determinan en gran parte la capacidad natatoria y de ataque a presas más veloces (Sibbing \& Nagelkerke 2000).

La última revisión bibliográfica publicada sobre las relaciones nivel trófico vs. talla informa que el $83 \%$ de las especies estudiadas (de un total de 271 especies marinas) exhiben una tendencia positiva al aumento del nivel trófico con el tamaño corporal, siendo el cambio en el nivel trófico promedio igual a un nivel a lo largo de su vida (Sweeting et al. 2012). En el presente trabajo se estudiaron cuatro especies que habitan el mismo ambiente (sistemas de arrecifes), y se ubican dentro de un rango trófico menor a un nivel trófico (Figura 4), por lo que se esperarían resultados similares y positivos para las cuatro especies. Sin embargo, la mitad de las especies presentó un cambio en el nivel trófico respecto del tamaño mientras que la otra mitad no. Estos resultados llaman la atención sobre la necesidad de contemplar la identidad de las especies en los modelos de cadena trófica estructurados por talla, no debiendo suponerse como generalizado el cambio alimentario talla-dependiente. Sin embargo, a partir de los resultados del presente trabajo, existe evidencia que sugiere que, a mayor amplitud de rango de crecimiento de un pez a lo largo de su vida, mayor es la probabilidad de aumentar de nivel trófico conforme crece. Esta evidencia apoyaría parcialmente los supuestos de los modelos tróficos estructurados por tallas.

Agradecimientos. Agradecemos a quienes colaboraron de diversas maneras haciendo posible la realización de este trabajo. A Irigoyen y L Venerus por sus aportes académicos y la discusión preliminar de este trabajo. G Trobbiani, P Fiorda y A Irigoyen por la ayuda prestada en la obtención de muestras. Los dibujos de los peces fueron realizados por E Vera. El trabajo fue financiado por los proyectos de la ANPCyT PICT 2008-1433 y PICT 2010-2461.

\section{BIBLIOGRAFÍA}

Albouy, C; F Guilhaumon; S Villéger; M Mouchet; L Mercier; JM Culioli; JA Tomasini; Le Loc'H F \& D Mouillot. 2011. Predicting trophic guild and diet overlap from functional traits: Statistics, opportunities and limitations for marine ecology. Mar. Ecol. Prog. Ser., 436: 7-28.

BACHILlER, E \& X IrIGOIEN. 2013. Allometric relations and consequences for feeding in small pelagic fish in the Bay of Biscay. ICES J Mar. Sci., 70:232-243.

BLyTh-SKYrME, RE; MJ KaISER; JG HiddinK; G EdWARdS-JONES \& PJB HART. 2006. Conservation benefits of temperate marine protected areas: Variation among fish species. Conserv. Biol., 20:811-820.

Boecklen, W; C Yarnes; B CoOK \& A James. 2011. On the Use of Stable Isotopes in Trophic Ecology. Annu. Rev. Ecol. Syst., 42:411-440

BRANKEVICH, A; A Roux \& R BASTIDA. 1990. Relevamiento de un banco de besugo (Spagrus pagrus) en la plataforma bonaerense. características fisiográficas generales y aspectos ecológicos preliminares. Frente Marítimo, 7:75-86.

Carabel, S; E Godínez-Domínguez; P Verísimo; L FernándeZ \& J FREIRE. 2006 An assessment of sample processing methods for stable isotope analyses of marine food webs. J. Exp. Mar. Biol. Ecol., 336:254-261.

Cohen, JE; SL Pimm; P Yodzis \& J SALdAnA. 1993. Body sizes of animal predators and animal prey in food webs. $J$. Anim. Ecol., 62:67-78.

Cousseau, MB \& R Perrota. 2000. Peces marinos de Argentina. Biología, Distribución y Pesca. Instituto de Investigación y Desarrollo Pesquero. Mar del Plata, Argentina.

Crawley, M.J. 2007. The R Book. J. Willey.

DAVENPORT, S \& NJ BAX. 2002. A trophic study of a marine ecosystem of southeastern Australia using stable isotopes of carbon and nitrogen. Can. J. Fish. Aquat. Sci., 59:514-530. 
David, GS; R Coutinho; I Quagio-Grassiotto \& JR Verani. 2005. The reproductive biology of Diplodus argenteus (Sparidae) in the coastal upwelling system of Cabo Frio, Rio de Janeiro, Brazil. Afr. J. Mar. Sci., 27:439-447.

DeNiro, M J \& S EPSTeIN. 1978. Influence of diet on the distribution of stable carbon isotopes in animals. Geochim. Cosmochim., 42:495-506.

Deudero S; J Pinnegar; NVC Polunin; G Morey; B Morales NIN. 2004. Spatial variation and ontogenic shifts in the isotopic composition of Mediterranean littoral fishes. Mar. Biol., 145:971-981.

FRANCE, RL. 1995. Carbon-13 enrichment in benthic compared to planktonic algae: foodweb implications. Mar. Ecol. Prog. Ser., 124:307-312.

FrY, B. 2006. Stable isotope Ecology. Springer Science+Business Media.

Froese, R \& D Pauly. 2013. FishBase. World Wide Web electronic publication. www.fishbase.org

Galván, D; L Venerus; A Irigoyen; AM Parma \& A GoszTONYI. 2005. Extension of the distributional range of the silver porgy, Diplodus argenteus (Valenciennes 1830), and the red porgy, Pagrus pagrus (Linnaeus 1758) (Sparidae) in northern Patagonia, south-western Atlantic. J. Appl. Ichthyol., 21:444-447.

Galván, DE; CJ Sweeting \& WDK Reid. 2010. Power of stable isotope techniques to detect size-based feeding in marine fishes. Mar. Ecol. Prog. Ser., 407:271-278.

Galván, DE; LA Venerus \& AJ Irigoyen. 2009. The Reeffish Fauna of the Northern Patagonian Gulfs, Argentina, Southwestern Atlantic. TOFishSJ., 2:25-31.

Goldstein, HE \& MB Cousseau. 1987. Estudios sobre el régimen alimentario del mero (Acanthistius brasilianus) y su relación con la características morfométricas del sistema digestivo (Pisces, Fam. Serranidae). Rev. Inv. Des. Pesq., 7:85-104.

Greenwood, NDW; CJ Sweeting \& NVC Polunin. 2010. Elucidating the trophodynamics of four coral reef fishes of the Solomon Islands using $\delta^{15} \mathrm{~N}$ and $\delta^{13} \mathrm{C}$. Coral Reefs, 29:785-792.

Gunderson, D; AM PARMA; R Hilborn; J COPE; D FluHARTY; ET AL. 2008. The Challenge of Managing Nearshore Rocky Reef Resources. Fisheries, 33:172-180.

Huntsman, G. 1996. Pagrus pagrus. In: IUCN 2012. IUCN Red List Of Threatened Species. Versión 2012.1. www.iucnredlist.org.

Irigoyen, AJ \& DE Galván. 2010. Peces de Arrecifes Argentinos. Proyecto Arrecife - CENPAT. Puerto Madryn, Argentina.

Jackson, AL; R Inger; A Parnell \& S Bearhop. 2011. Comparing isotopic niche widths among and within communities: SIBER - Stable Isotope Bayesian Ellipses in R. J Anim., Ecol. 80:595-602.

Jennings, S; J Oliveira \& KJ Warr. 2007. Measurement of body size and abundance in tests of macroecological and food web theory. J. Anim. Ecol., 76:72-82.

Jennings, S; JK Pinnegar; NVC Polunin \& TW Boon. 2001. Weak cross-species relationships between body size and trophic level belie powerful size-based trophic structuring in fish communities. J. Anim. Ecol., 70:934-944.

Jennings, S; JK Pinnegar; NVC Polunin \& KJ Warr. 2002. Linking size-based and trophic analyses of benthic community structure. Mar. Ecol. Prog. Ser., 226:77-85.

Jiao, Y; Y Chen; D SChNeider \& J WroblewsKi. 2004. A simulation study of impacts of error structure on modeling stock-recruitment data using generalized linear models. Can J. Fish. Aquat. Sci., 61:122-133.

Karpouzi, VS \& KI Stergiou. 2003. The relationships between mouth size and shape and body length for 18 species of marine fishes and their trophic implications. J. Fish. Biol., 62: 1353-1365.

Logan, JM; TD JaRdine; TJ Miller; SE BunN; RA CunJaK \& ME Lutcavage. 2008. Lipid corrections in carbon and nitrogen stable isotope analyses: Comparison of chemical extraction and modelling methods. J. Anim. Ecol., 77:838-846.

PinNegar, J \& NVC Polunin. 1999. Differential fractionation of $\delta^{13} \mathrm{C}$ and $\delta^{15} \mathrm{~N}$ among fish tissues: implications for the study of trophic interactions. Funct. Ecol., 13:225-231.

Polis, GA \& DR Strong. 1996. Food Web Complexity and Community Dynamics. Amer. Nat., 147:813-846.

Post, D. 2002. Using Stable Isotopes to Estimate Trophic Position: Models, Methods, and Assumptions. Ecology, 83:703-718.

Post, D; M Pace \& N Hairston JR. 2000. Ecosystem size determines food-chain length in lakes. Nature, 405: 1047-1049.

R Development Core Team. 2010. R: a language and environment for statistical computing. R Foundation for Statistical Computing

Reid, WDK; S Clarke; MA Collins \& M Belchier. 2007. Distribution and ecology of Chaenocephalus aceratus (Channichthyidae) around south Georgia and Shag Rocks (Southern Ocean). Polar Biol., 30:1523-1533.

Stal, J; L Pinl \& H Wennhage. 2007. Food utilization by coastal fish assemblages in rocky and soft bottoms on the Swedish west coast: Inference for identification of essential fish habitats. Est. Coast. Shelf Sci., 71:593-607.

Scharf, FS; Juanes F \& RA Rountree. 2000. Predator size-prey size relationships of marine fish predators: interspecific variation and effects of ontogeny and body size on trophic-niche breadth. Mar. Ecol. Prog. Ser., 208:229-248.

SibBING, FA \& LAJ NAGELKERKE . 2000. Resource partitioning by Lake Tana barbs predicted from fish morphometrics and prey characteristics. Rev. Fish Biol. Fish., 10:393-437.

Stergiou KI \& VS Karpouzi. 2002. Feeding habits and trophic levels of Mediterranean fish. Rev. Fish Biol. Fish., 11:217-254.

Sweeting, CJ; JP Barry; C Barnes; NVC Polunin \& S JENNINGS. 2007a. Effects of body size and environment on diet-tissue $\delta^{15} \mathrm{~N}$ fractionation in fishes. J. Exp. Mar. Biol. Ecol., 340:1-10.

SweEting, CJ; JP Barry; NVC Polunin \& S JenNings. 2007b. Effects of body size and environment on diet-tissue $\delta^{3} \mathrm{C}$ fractionation in fishes. J. Exp. Mar. Biol. Ecol., 352:165-176.

SweEting, CJ; NVC Polunin \& S Jennings. 2006. Effects of chemical lipid extraction and arithmetic lipid correction on stable isotope ratios of fish tissues. Rapid. Commun. Mass. Spectrom., 20: 595-601.

SWEETING, CJ; WDK REID \& DE GALVÁn. 2012. Exploring the ubiquity and form of size based feeding in marine fishes. Pp. 163 In: Book of Abstracts 6th Word Fisheries Congress. 